\section{Manejo de hemorragia asociada a anticoagulantes orales directos: estado actual de las estrategias de reversión}

ANDRÉS ENRÍQUEZ ${ }^{1,2}$, ADRIAN BARANCHUK ${ }^{2}$, RAMÓN CORBALÁN $^{3}$

Direct oral anticoagulants (DOACs), including the direct thrombin inhibitor dabigatran and the direct factor Xa inhibitors rivaroxaban, apixaban and edoxaban have at least comparable efficacy as vitamin K antagonists along with a better safety profile, reflected by a lower incidence of intracranial hemorrhage. Specific reversal agents have been developed in recent years. Namely, idarucizumab, a specific antidote for dabigatran, is currently approved in most countries. Andexanet, which reverses factor Xa inhibitors, has been recently approved by the FDA, and ciraparantag, a universal antidote targeted to reverse all DOACs, is still under investigation. In this review we provide an update on the pharmacology of DOACs, the risk of hemorrhagic complications associated with their use, the measurement of their anticoagulant effect and the reversal strategies in case of DOAC-associated bleeding.

(Rev Med Chile 2019; 147: 73-82)

Key words: Anticoagulants; Hemorrhage; Risk Factors.

E 1 desarrollo de los anticoagulantes orales directos o DOACs (direct oral anticoagulants) para el manejo de la enfermedad tromboembólica es una historia de éxito como pocas. En el transcurso de la última década han reemplazado progresivamente a los antagonistas de la vitamina $\mathrm{K}(\mathrm{AVK})$, que fueron la única clase disponible de anticoagulantes orales por casi 6 décadas. Los DOACs actúan por inhibición directa de la trombina (dabigatrán) o el factor Xa (fXa) (rivaroxabán, apixabán y edoxabán). Son tan eficaces como la warfarina para tromboprofilaxis en pacientes con fibrilación auricular (FA) y para la prevención y tratamiento de la enfermedad tromboembólica venosa, y se asocian a un riesgo menor o comparable de sangrado mayor ${ }^{1,2}$. En comparación con los AVK, los DOACs tienen un efecto terapéutico más predecible, no requieren
'Departamento de Cardiología,

Hospital Guillermo Grant

Benavente. Concepción, Chile.

2Division of Cardiology, Queen's

University, Kingston, Ontario.

Canada.

${ }^{3}$ División de Enfermedades

Cardiovasculares, Hospital Clínico

Pontificia Universidad Católica de

Chile. Santiago, Chile.

Los autores declaran no tener conflictos de interés.

Trabajo no recibió finaciamiento.

Recibido el 12 de septiembre de 2018, aceptado el 16 de

noviembre de 2018.

Correspondencia a:

Dr. Andrés Enríquez

Heart Rhythm Service

Kingston General Hospital

Fax: + 1-613-5481387

Tel: + 1-613-5496666 ext 6578

Andres.Enriquez@kingstonhsc.ca

monitoreo del efecto anticoagulante, tienen menos interacciones con otros fármacos y no requieren restricción de alimentos que contienen vitamina K.

Una de las principales preocupaciones desde la introducción de los DOACs ha sido la ausencia de agentes específicos para revertir su efecto en caso de hemorragia graves o necesidad de cirugía de urgencia. Si bien estos no estaban disponibles al tiempo en que se desarrollaron los estudios clásicos que establecieron el beneficio de los DOACs, las consecuencias de sangrados mayores asociados a DOACs no fueron peores que en pacientes tratados con warfarina ${ }^{3-6}$. El idarucizumab, antídoto del dabigatrán, está disponible en Chile desde el año 2016 y el andexanet, primer antídoto contra inhibidores del fXa, ha sido recientemente aprobado por la Food and Drug Administration (FDA) ${ }^{7,8}$. 
En esta revisión se aborda el manejo de las complicaciones hemorrágicas secundarias al uso de DOACs y el actual estado de desarrollo de los antídotos.

\section{Farmacología de los DOACs}

Los DOACs disponibles comercialmente incluyen el inhibidor directo de la trombina dabigatrán (Pradaxa; Boehringer Ingelheim) y los inhibidores del fXa rivaroxabán (Xarelto; Johnson and Johnson/Bayer HealthCare), apixabán (Eliquis; Bristol-Myers Squibb/Pfizer) y edoxabán (Savaysa o Lixiana; Daiichi Sankyo) (Tabla 1). En base a la evidencia, son 3 los escenarios clínicos en los cuales los DOACs tendrían indicación: prevención de accidente vascular cerebral (AVC) en FA no valvular, tratamiento de tromboembolismo venoso, y prevención de tromboembolismo venoso postcirugía de reemplazo de cadera o rodilla (edoxabán aprobado solo en Japón para esta indicación). Un cuarto inhibidor del fXa, el betrixabán (Bevyxxa; Portola Pharmaceuticals), ha sido aprobado en Estados Unidos de Norteamérica solo para la prevención de tromboembolismo venoso en pacientes con enfermedad médica aguda ${ }^{9}$ y no se incluirá en esta revisión.

Desde un punto de vista farmacocinético, los DOACs se caracterizan por un rápido inicio de acción, con un efecto máximo 1-4 h postingestión ${ }^{10,11}$, y tienen una vida media $(\mathrm{Vm})$ más corta que los AVK (10-14 h versus 36-42 h). Todos los DOACs son eliminados en parte por el riñón: $80 \%$ en caso del dabigatrán, $33 \%$ rivaroxabán, $25 \%$ apixabán y $50 \%$ edoxabán. Esto es relevante, ya que pacientes con hemorragia pueden cursar con injuria renal aguda que puede retrasar su clearence. Un potencial mecanismo de interacción de los DOACs está dado por la resecreción a través de una glicoproteína $\mathrm{P}$ (gp-P) después de su absorción intestinal ${ }^{12}$. De este modo, la coadministración de inhibidores de la gp-P (amiodarona, verapamil, diltiazem, digoxina) puede resultar en

Tabla 1. Propiedades farmacocinéticas de los DOACs

\begin{tabular}{|c|c|c|c|c|}
\hline & Dabigatrán & Rivaroxabán & Apixabán & Edoxabán \\
\hline $\begin{array}{l}\text { Dosis } \\
\text { - FA } \\
\text { - Profilaxis TVP/EP } \\
\text { - Tratamiento de TVP/EP }\end{array}$ & $\begin{array}{l}150 \mathrm{mg} \text { BID } \\
150 \mathrm{mg} \text { BID } \\
150 \mathrm{mg} \text { BID }\end{array}$ & $\begin{array}{l}20 \text { mg QD } \\
10 \text { mg QD } \\
15 \text { mg BID por } 21 \mathrm{~d} \\
\text { luego } 20 \mathrm{mg} \text { QD }\end{array}$ & $\begin{array}{l}5 \mathrm{mg} \text { BID } \\
2,5 \mathrm{mg} \text { BID } \\
10 \mathrm{mg} \text { BID por } 7 \mathrm{~d} \\
\text { luego } 5 \mathrm{mg} \mathrm{BID}\end{array}$ & $\begin{array}{l}60 \text { mg QD } \\
30 \text { mg QD } \\
60 \text { mg QD después de te- } \\
\text { rapia inicial con heparina }\end{array}$ \\
\hline Peso molecular (Da) & 628 & 436 & 460 & 548 \\
\hline Target & $\|$ & $\mathrm{Xa}$ & $\mathrm{Xa}$ & $\mathrm{Xa}$ \\
\hline Biodisponibilidad (\%) & 6 & $63-79$ & 66 & 50 \\
\hline Efecto máximo (h) & $1-3$ & $2-4$ & $1-2$ & $1-2$ \\
\hline Vida media (h) & $12-17$ & $7-13$ & $8-15$ & $9-11$ \\
\hline Unión a proteínas (\%) & 35 & 95 & 87 & 54 \\
\hline Metabolismo & $\begin{array}{l}80 \% \text { renal } \\
20 \% \text { hepático }\end{array}$ & $\begin{array}{l}\text { 1/3 renal } \\
2 / 3 \text { hepático }\end{array}$ & $\begin{array}{l}25 \% \text { renal } \\
75 \% \text { fecal }\end{array}$ & $\begin{array}{l}35 \% \text { renal } \\
63 \% \text { hepático }\end{array}$ \\
\hline Interacciones & $\begin{array}{l}\text { Inhibores de la } \\
\text { gp-P }\end{array}$ & $\begin{array}{l}\text { Inhibidores de } \\
\text { CYP3A4 Inhibores de } \\
\text { la gp-P }\end{array}$ & $\begin{array}{l}\text { Inhibidores de } \\
\text { CYP3A4 Inhibores de } \\
\text { la gp-P }\end{array}$ & $\begin{array}{l}\text { Inhibidores de CYP3A4 } \\
\text { Inhibores de la gp-P }\end{array}$ \\
\hline Indicaciones aprobadas & $\begin{array}{l}\text { - Prevención de } \\
\text { AVC en FA no } \\
\text { valvular } \\
\text { - TEV (profilaxis y } \\
\text { tratamiento) }\end{array}$ & $\begin{array}{l}\text { - Prevención de AVC } \\
\text { en FA no valvular } \\
\text { - TEV (profilaxis y } \\
\text { tratamiento) }\end{array}$ & $\begin{array}{l}\text { - Prevención de AVC } \\
\text { en FA no valvular } \\
\text { - TEV (profilaxis y } \\
\text { tratamiento) }\end{array}$ & $\begin{array}{l}\text { - Prevención de AVC en } \\
\text { FA no valvular } \\
\text { - TVE (tratamiento, en } \\
\text { Japón también para } \\
\text { profilaxis) }\end{array}$ \\
\hline
\end{tabular}

*FA: fibrilación auricular; TVP: trombosis venosa profunda; EP: embolia pulmonar; BID: cada 12 h; QD: una vez al dia; gp-P: glicoproteína P; CYP3A4: citocromo P450 3A4; AVC: accidente vascular cerebral; TEV: tromboembolismo venoso. 
un aumento de los niveles plasmáticos de droga ${ }^{13}$. Asimismo, el citocromo P450 3A4 (CYP3A4) está involucrado en la eliminación hepática del rivaroxabán y apixabán, lo que puede ser una fuente de interacción ante coadministración de inhibidores del CYP3A4 (atorvastatina, azoles, eritromicina $)^{12,13}$.

\section{Riesgo de sangrado}

El sangrado es el principal efecto adverso de los anticoagulantes orales y la hemorragia intracraneana (HIC) su complicación más temida. En pacientes tratados con AVK el riesgo de sangrado mayor se estima en $1-2 \%$ anual ${ }^{14}$, pero la incidencia es más alta ( 3-4\%) en estudios de la comunidad, que típicamente incluyen pacientes mayores, con más comorbilidades y con peor manejo de las dosis ${ }^{15,16}$. En comparación con warfarina, el riesgo de sangrado mayor es significativamente reducido por dabigatrán en dosis de $110 \mathrm{mg}$ cada $12 \mathrm{~h}$, apixabán y edoxabán, mientras que es similar a warfarina con dabigatrán en dosis de $150 \mathrm{mg}$ cada 12 h y rivaroxabán ${ }^{3-6}$.

Todos los DOACs se asocian a reducción significativa de la $\mathrm{HIC}$, con hazard ratios que oscilan entre 0,31 y $0,64^{3-6}$. Datos con dabigatrán y apixabán también muestran que las consecuencias de la HIC son mejores con DOACs ${ }^{17,18}$. La tasa de hemorragia gastrointestinal, por otro lado, es significativamente mayor con dabigatrán en dosis de $150 \mathrm{mg}$ cada $12 \mathrm{~h}$, rivaroxabán y edoxabán $60 \mathrm{mg}$, es similar a la de warfarina con apixabán y dabigatrán en dosis de $110 \mathrm{mg}$ cada $12 \mathrm{~h}$ y es menor a la de warfarina con edoxabán $30 \mathrm{mg}^{3-6}$. Grandes estudios postmarketing con diversos DOACs han confirmado la seguridad de estas drogas en el mundo real. Un metaanálisis que incluyó 28 estudios poblacionales de dabigatrán, rivaroxabán y apixabán confirmaron un menor riesgo de HIC con las 3 drogas, mayor riesgo de hemorragia gastrointestinal con dabigatrán y rivaroxabán, y menor riesgo de hemorragia gastrointestinal con apixabán ${ }^{19}$.

\section{Medición del efecto anticoagulante}

La determinación del efecto anticoagulante puede ser útil en situaciones como sobredosis, hemorragia o cirugía de urgencia.
Los niveles de dabigatrán pueden medirse directamente mediante cromatografía líquida acoplada a espectrometría de masas en tándem, que es considerado el gold standard, pero solo está disponible en centros especializados ${ }^{20}$. Los tests más usados en la práctica clínica son ensayos de coagulación funcionales como el test de ecarina $\mathrm{y}$ el test de trombina diluida Hemoclot ${ }^{\circledR}$, que reflejan la actividad de la trombina en el plasma y muestran una correlación lineal con los niveles de dabigatrán ${ }^{20,21}$. Entre los exámenes de coagulación de rutina, el tiempo de tromboplastina parcial activado (TTPa) provee una estimación cualitativa del efecto anticoagulante de dabigatrán; un valor normal, en general, excluye niveles significativos de la droga si un reactante sensible es usado, mientras que un TTPa prolongado sugiere niveles en rango terapéutico o supraterapéutico ${ }^{11}$.

En el caso de los inhibidores del fXa, el indicador de laboratorio más específico es la medición de la actividad anti-fXa por ensayos cromogénicos, que proveen una determinación cuantitativa de los niveles de droga ${ }^{20,22-24}$. El tiempo de protrombina (TP) permite una determinación cualitativa del efecto de rivaroxabán y en menor medida del edoxabán, pero es poco útil en caso del apixabán ${ }^{23}$. Un TP prolongado sugiere niveles terapéuticos o supraterapéuticos de las drogas, sin embargo, la sensibilidad depende del reactante utilizado, por lo que un valor normal no siempre excluye niveles clínicamente significativos.

\section{Medidas para la reversión de los DOACs}

\section{a. Agentes hemostáticos no específicos}

Los agentes hemostáticos que han sido estudiados incluyen el concentrado de complejo de protrombina (CCP) y factor VIIa recombinante (rFVIIa).

\section{a.1. $C C P$}

Es el agente actualmente más usado en caso de sangrado mayor asociado a inhibidores del $\mathrm{fXa}$. El CCP consiste en complejos liofilizados de factores de coagulación vitamina $\mathrm{K}$-dependientes y existen 3 productos disponibles comercialmente: (a) CCP de 3 factores (CCP-3): Contiene factores II, IX y X (Profilnine ${ }^{\circledR}$ SD, Bebulin ${ }^{\circledR}$ VH); (b) CCP de 4 factores (CCP-4): Contiene factores II, VII, IX y X $\left(\right.$ Octaplex $^{\circledR}$, Beriplex $^{\circledR}$, Cofact $^{\circledR}$, Kanokad $^{\circledR}$, 
Kaskadil $^{\circledR}$ ); y CCP activado (CCPA): Contiene factores de coagulación en su forma activada $\left(\text { FEIBA }^{\circledR}\right)^{7}$. La evidencia de la eficacia del CCP deriva de estudios de laboratorio ex-vivo e in-vivo (modelos animales), estudios en voluntarios sanos usando tests de coagulación y también datos limitados en pacientes con hemorragia asociada a DOACs. El uso de CCP se asocia a una incidencia de eventos tromboembólicos de $0,7 \%$ con CCP-3 y $1,8 \%$ con CCP- $4^{25}$.

Estudios en voluntarios sanos tratados con rivaroxabán, apixabán y edoxabán han demostrado que la administración de CCP-3 o CCP-4 restaura el TP y la generación de trombina a niveles normales o casi normales ${ }^{26-28}$. El primer estudio in-vivo en humanos fue realizado por Eerenberg con $\mathrm{CCP}-4^{26}$. En este estudio aleatorizado, 12 voluntarios sanos de sexo masculino recibieron dabigatrán $150 \mathrm{mg}$ cada 12 h o rivaroxabán 20 $\mathrm{mg}$ al día por $2,5 \mathrm{~d}$, seguido por un bolo de CCP4 de $50 \mathrm{UI} / \mathrm{kg}$ o un volumen similar de solución salina. A continuación, los grupos fueron cruzados y recibieron el otro anticoagulante siguiendo el mismo protocolo. CCP-4 logró normalización de los tests de coagulación postadministración de rivaroxabán, pero no fue efectivo para revertir el efecto anticoagulante del dabigatrán.

En un estudio de cohorte prospectivo, Majeed evaluó la eficacia de CCP-4 para el manejo de sangrado mayor en 84 pacientes recibiendo rivaroxabán o apixabán, incluyendo HIC en 59 pacientes y hemorragia gastrointestinal en 13 pacientes $^{29}$. CCP-4 fue efectivo en $69,1 \%$ de los pacientes, pero solo en $27 \%$ de los pacientes con HIC. Dos pacientes sufrieron un AVC isquémico y 27 pacientes murieron a 30 días de seguimiento.

Un estudio retrospectivo reportó los resultados del uso de FEIBA (20 UI/Kg) en 11 pacientes con HIC asociada a inhibidores del $\mathrm{fXa}^{30}$. El 55\% de los pacientes mostró estabilización del sangrado en la tomografía computada de control, mientras que 36\% mostró expansión del hematoma. Dos pacientes desarrollaron eventos trombóticos, incluyendo AVC isquémico en un caso y tromboembolismo pulmonar en otro.

\section{a.2. rFVIIa}

El rFVIIa actúa por unión al factor tisular en sitios de injuria vascular e inducción de la generación de trombina. Ha revelado potencial para normalizar los parámetros de coagulación in-vitro $y$ en modelos animales ${ }^{31}$, pero el efecto sobre la magnitud del sangrado es variable. El uso de rFVIIa ha sido reportado para manejar el sangrado postcirugía cardíaca asociada a dabigatrán ${ }^{32}$, pero no existen estudios sistemáticos en seres humanos. $\mathrm{Al}$ igual que con CCP, su uso se ha asociado a un riesgo aumentado de tromboembolismo.

\section{b. Antídotos específicos}

Tres antídotos específicos han sido desarrollados para revertir el efecto anticoagulante de los DOACs (Tabla 2, Figura 1). El idarucizumab (Praxbind; Boehringer Ingelheim), antídoto especifico del dabigatrán, se encuentra actualmente disponible en muchos países, incluido Chile. El andexanet alfa (Andexxa; Portola Pharmaceuticals), destinado a revertir el efecto de los inhibidores del fXa y las heparinas, fue aprobado por la FDA en mayo de 2018 y se encuentra en evaluación por la Asociación Europea de Medicamentos. Por último, el ciraparantag, que tendría acción sobre todos los DOACs y heparinas, se encuentra en evaluación fase II.

\section{b.1. Idarucizumab}

Es un antídoto específico de dabigatrán desarrollado por Boehringer Ingelheim y aprobado por la FDA en octubre de 2015. Es un fragmento Fab de anticuerpo monoclonal humanizado que se une al dabigatrán con una afinidad 350 veces superior a la trombina, neutralizando su activi$\mathrm{dad}^{33}$. Después de su administración endovenosa tiene una $\mathrm{Vm}$ de aproximadamente $45 \mathrm{~min}$ en pacientes con función renal normal, pero una $\mathrm{Vm}$ más prolongada en presencia de alteración de la función renal.

Estudios in vivo en ratas revelan que niveles de dabigatrán de $200 \mathrm{ng} / \mathrm{ml}$ son neutralizados dentro de 1 min luego de la administración de un bolo intravenoso de idarucizumab ${ }^{33}$. Asimismo, estudios fase 1 en voluntarios jóvenes sanos, en individuos de 65-80 años de edad y en individuos de 45-80 años con deterioro leve a moderado de la función renal demostraron que la administración de idarucizmuab produjo reversión inmediata y completa del efecto anticoagulante del dabigatrán, sin efectos adversos significativos ${ }^{34-36}$.

El RE-VERSE AD es un estudio fase 3 prospectivo que incluyó 503 pacientes tratados con dabigatrán que presentaron un sangrado grave (grupo A) o que requirieron una cirugía o proce- 
Tabla 2. Antídotos específicos para DOACs

\begin{tabular}{|c|c|c|c|}
\hline Agente & $\begin{array}{l}\text { Idarucizumab } \\
\text { (Boehringer Ingelheim) }\end{array}$ & $\begin{array}{l}\text { Andexanet } \\
\text { (Portola Pharmaceuticals) }\end{array}$ & $\begin{array}{l}\text { Ciraparantag } \\
\text { (Perosphere) }\end{array}$ \\
\hline $\begin{array}{l}\text { Drogas } \\
\text { revertidas }\end{array}$ & Dabigatrán & $\begin{array}{l}\text { Inhibidores del FXa (rivaroxabán, } \\
\text { apixabán, edoxabán, betrixabán) }\end{array}$ & $\begin{array}{l}\text { Dabigatrán, inhibidores del FXa } \\
\text { (rivaroxabán, apixabán, edoxa- } \\
\text { bán, betrixabán), fondaparinux, } \\
\text { heparina }\end{array}$ \\
\hline Estructura & $\begin{array}{l}\text { Fragmento de anticuerpo hu- } \\
\text { manizado }\end{array}$ & $\begin{array}{l}\text { FXa recombinante, catalítica- } \\
\text { mente inactivo }\end{array}$ & Molécula sintética catiónica \\
\hline Mecanismo & $\begin{array}{l}\text { Unión no competitiva a dabiga- } \\
\text { trán con una afinidad } 350 \text { veces } \\
\text { mayor a la trombina }\end{array}$ & $\begin{array}{l}\text { Unión competitiva a los inhibi- } \\
\text { dores del FXa }\end{array}$ & $\begin{array}{l}\text { Unión a través de uniones no } \\
\text { covalentes }\end{array}$ \\
\hline $\begin{array}{l}\text { Forma de } \\
\text { administración }\end{array}$ & $\begin{array}{l}5,0 \mathrm{~g} \text { IV en } 2 \text { dosis de } 2,5 \mathrm{~g} \\
\text { IV (separadas por no más de } \\
15 \mathrm{~min} \text { ) }\end{array}$ & $\begin{array}{l}\text { Rivaroxabán o edoxabán: } \\
800 \text { mg IV seguido de } 8 \text { mg/Kg } \\
\text { por } 2 \text { h; apixabán: } 400 \text { mg IV } \\
\text { seguido de } 4 \text { mg/Kg por } 2 \text { h }\end{array}$ & $\begin{array}{l}\text { Infusión IV (100-300 mg en } \\
\text { estudios clínicos) }\end{array}$ \\
\hline Vida media & Bifásica (Inicial 45 min, final 10 h) & $5-7 \mathrm{~h}$ & $1,5 \mathrm{~h}$ \\
\hline Disponibilidad & $\begin{array}{l}\text { Aprobado en la mayoría de los } \\
\text { países }\end{array}$ & $\begin{array}{l}\text { Aprobado por la FDA y en eva- } \\
\text { luación por la Agencia Europea } \\
\text { de Medicamentos }\end{array}$ & $\begin{array}{l}\text { No enviado aún a agencias } \\
\text { regulatorias }\end{array}$ \\
\hline \multicolumn{4}{|l|}{ Estudios clínicos } \\
\hline $\begin{array}{l}\text { Fase I-II } \\
\text { Fase III }\end{array}$ & $\begin{array}{l}\text { - Jóvenes sanos, individuos de } \\
65-80 \text { a, individuos de } 45-80 \\
\text { a con deterioro de función } \\
\text { renal } \\
\text { - Sangrado no controlado o } \\
\text { de riesgo vital y cirugía o } \\
\text { procedimiento de urgencia } \\
\text { (RE-VERSE AD) }\end{array}$ & $\begin{array}{l}\text { - Sangrado agudo mayor aso- } \\
\text { ciado a rivaroxabán, apixa- } \\
\text { bán, edoxabán y enoxaparina } \\
\text { (ANNEXA-4) }\end{array}$ & - Voluntarios sanos \\
\hline
\end{tabular}

dimiento de urgencia (grupo B) ${ }^{37}$. Idarucizumab fue administrado como 2 bolos de 2,5 $\mathrm{g}$ (total de $5,0 \mathrm{~g})$ administrados a lo largo de 5-10 min. En el grupo A $(\mathrm{n}=301), 45,5 \%$ se presentaron con hemorragia gastrointestinal, $32,6 \%$ con HIC y $25,9 \%$ con sangrado asociado a trauma. En el grupo B $(\mathrm{n}=202) 98 \%$ fue sometido a cirugía, incluyendo cirugía abdominal en $24,3 \%$, ortopédica en $20,3 \%$, cardiovascular en $18,3 \%$, cerebral en $8,4 \%$, hepatobiliar en 6,9\% y trauma de tórax en 6,9\%. Más de 95\% de los pacientes estaba recibiendo dabigatrán para profilaxis de AVC en FA y la edad promedio fue de 78 años. El 43,3\% de los pacientes tenía un clearance de creatinina $<50 \mathrm{ml} / \mathrm{min}$, medido por fórmula de Cockcroft-Gault. El end point primario fue el porcentaje máximo de reversión del efecto anticoagulante dentro de $4 \mathrm{~h}$ de administración del idarucizumab y fue de $100 \%$, medido por test de ecarina y test de trombina diluida. En el grupo A, el tiempo medio al cese del sangrado fue de $2,5 \mathrm{~h}$. En el grupo B, el tiempo medio hasta el comienzo del procedimiento fue de $1,6 \mathrm{~h}$ y la hemostasia fue catagolada por el cirujano como normal en 93,4\% de los pacientes. La incidencia de eventos tromboembólicos a 90 días fue de 6,3\% en el grupo A y de $7,4 \%$ en el grupo B, mientras que la mortalidad fue de $18,8 \%$ y $18,9 \%$, respectivamente. No se reportaron reacciones de hipersensibilidad.

\section{b.2. Andexanet}

Es un fármaco inyectable desarrollado por Portola Pharmaceuticals como antídoto contra los inhibidores del fXa y que también es capaz de revertir el efecto anticoagulante de la heparina de bajo peso molecular y heparina no fraccionada. La droga es una forma recombinante, modificada, de fXa que se une con alta afinidad a los inhibidores del fXa, pero es catalíticamente inactiva ${ }^{38,39}$. 


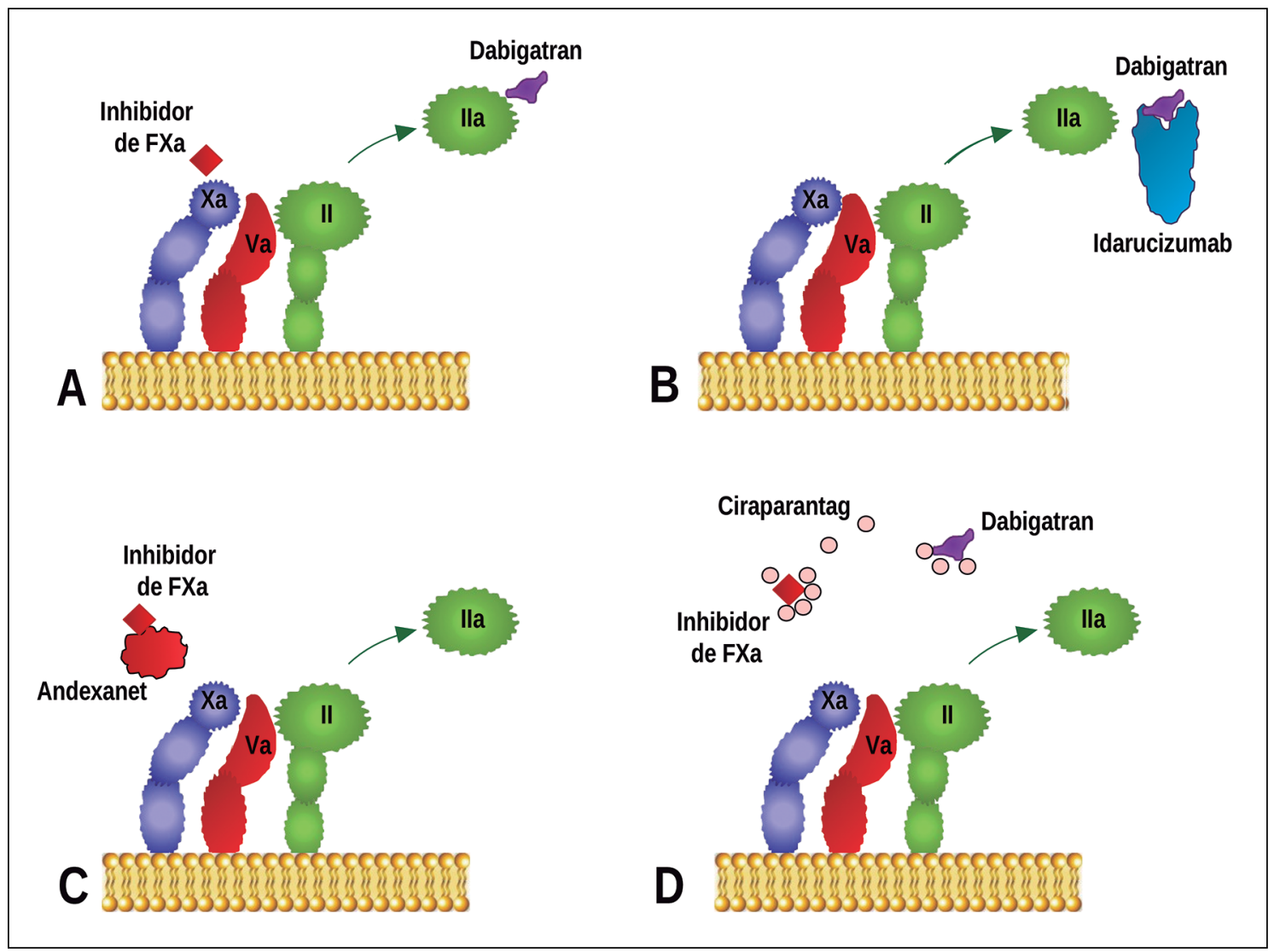

Figura 1. Mecanismo de los DOACs y sus antídotos. Panel A: El complejo protrombinasa, formado por los factores Xa y Va, cataliza la conversión de protrombina (II) en trombina (Ila). La trombina, a su vez, convierte el fibrinógeno en fibrina, responsable de la agregación plaquetaria. Los inhibidores del FXa y el dabigatrán ejercen su efecto anticoagulante por unión al FXa y a la trombina, respectivamente. Panel B: Idarucizumab es un fragmento de anticuerpo Fab humanizado que se une al dabigatrán, evitando su unión a la trombina y neutralizando su efecto anticoagulante. Panel C: El andexanet es un FXa recombinante modificado que carece de actividad catalítica. Al unirse a los inhibidores del FXa circulantes, el andexanet permite que el FXa convierta la protrombina en trombina, restaurando la cascada de la coagulación. Panel D: El ciraparantag es una molécula sintética pequeña que se une a varias drogas anticoagulantes a través de uniones no covalentes (puentes de hidrógeno e interacciones carga-carga), restaurando la actividad de los factores de coagulación bloqueados. Modificado con autorización de: Enriquez et al. $\left.{ }^{8}\right)$.

Estudios fase 2 en voluntarios sanos demostraron que el andexanet es capaz de revertir inmediatamente y en forma dosis-dependiente la actividad anti-fXa de rivaroxabán, apixabán y edoxabán ${ }^{40,41}$. En los estudios ANNEXA-A y ANNEXA-R, voluntarios sanos de 50-75 años recibieron una dosis estándar de apixabán y rivaroxabán hasta alcanzar el steady-state, seguido de la administración intravenosa de andexanet (bolo de $400 \mathrm{mg}$ para apixabán y $800 \mathrm{mg}$ para rivaroxabán $)^{40}$. La droga se asoció a reducción rápida y significativa de la actividad anti-fXa, medida por ensayo cromogénico, y de la concentración plasmática de apixabán y rivaroxabán libre dentro de 2 a 5 min de la administración intravenosa. Un aumento en ambas mediciones se observó a los 15 min de terminado el bolo, siendo necesaria una infusión de 2 h para lograr supresión sostenida de la actividad anticoagulante. No se reportó efectos adversos serios ni complicaciones trombóticas en los 101 pacientes que recibieron andexanet.

Un estudio prospectivo multicéntrico fase 3, el 
ANNEXA-4, se encuentra actualmente en desarrollo para evaluar el uso de andexanet en pacientes con sangrado mayor asociado a apixabán, rivaroxabán, edoxabán y enoxaparina. En este estudio, los pacientes que estaban recibiendo apixabán o que habían recibido rivaroxabán $>7 \mathrm{~h}$ antes de la administración de andexanet, recibieron un bolo de $400 \mathrm{mg}$ seguido de infusión de $480 \mathrm{mg}$ en $2 \mathrm{~h}$, mientras que los pacientes que estaban recibiendo edoxabán, enoxaparina o que habían recibido rivaroxabán $\leq 7 \mathrm{~h}$ antes de la administración de andexanet recibieron un bolo de $800 \mathrm{mg}$ seguido de infusión de $960 \mathrm{mg}$ en $2 \mathrm{~h}$. Los 2 objetivos primarios fueron el porcentaje de cambio de la actividad anti-fXa y la tasa de hemostasia excelente o buena a las $12 \mathrm{~h}$ de terminada la infusión de andexanet. Los resultados de los primeros 67 pacientes enrolados en el estudio han sido publicados $^{42}$. De estos, 32 pacientes estaban recibiendo rivaroxabán, 31 estaban recibiendo apixabán y 4 estaban recibiendo enoxaparina. La edad promedio de los pacientes era de 77 años y el tracto gastrointestinal fue el sitio primario de sangrado más frecuente (49\%), seguido de HIC en segundo lugar $(42 \%)$. Solo los 47 pacientes con una actividad anti-fXa basal $\geq 75 \mathrm{ng} / \mathrm{ml}$ fueron incluidos en el análisis de eficacia. Después de la administración del bolo, la actividad anti-fXa disminuyó en $89 \%$ en los pacientes recibiendo rivaroxabán y en 93\% en los pacientes recibiendo apixabán. A las $12 \mathrm{~h}$ de terminada la infusión de andexanet, la hemostasia fue catalogada como excelente o buena en 37 de los 47 pacientes incluidos en el análisis de eficacia (79\%). Eventos trombóticos ocurrieron en 18\% de los pacientes a los $30 \mathrm{~d}$ de seguimiento.

\section{b.3. Ciraparantag}

Es un antídoto de amplio espectro desarrollado por Perosphere Pharmaceuticals. Es una pequeña molécula catiónica sintética, hidrosoluble, que se une a la heparina no fraccionada, heparina de bajo peso molecular, fondaparinux, dabigatrán $\mathrm{y}$ a los inhibidores del $\mathrm{fXa}$ a través de puentes de hidrógeno y enlaces iónicos ${ }^{43}$. En ensayos ex-vivo y en modelos animales de sangrado el ciraparantag ha demostrado reversión del efecto anticoagulante del dabigatrán y de los inhibidores del fXa rivaroxabán, apixabán y edoxabán ${ }^{43-45}$. Potenciales efectos adversos incluyen flushing y disgeusia.

Un estudio fase I/II en 80 voluntarios sanos evaluó el efecto de una dosis intravenosa de cira- parantag solo y postadministración de edoxabán ${ }^{46}$. La droga produjo normalización del tiempo de coagulación dentro de 10 min o menos y el efecto fue sostenido por $24 \mathrm{~h}$. El ciraparantag no mostró evidencia de actividad procoagulante, determinada por tiempo de coagulación de sangre total, dímero $\mathrm{D}$, fragmento de protrombina 1,2 e inhibidor de la vía del factor tisular. Otro estudio se encuentra en marcha para evaluar la utilidad de ciraparantag para revertir el efecto de rivaroxabán ${ }^{47}$.

\section{Algoritmo de manejo en caso de hemorragia}

Recomendaciones para el enfrentamiento de las complicaciones hemorrágicas asociadas a DOACs han sido publicadas por sociedades médicas internacionales ${ }^{11,12,48}$. En la Figura 2 se observa un algoritmo que resume las medidas sugeridas según la magnitud del sangrado.

La discontinuación transitoria de la droga, el control de la fuente de sangrado y medidas de soporte hemodinámico, tales como reposición de fluidos y tranfusión de hemoderivados, constituyen las bases del manejo. Las medidas locales de hemostasia incluyen compresión mecánica, cirugía, terapia endoscópica, embolización y otras intervenciones para lograr control del sangrado. Pacientes con hemorragia de riesgo vital o hemorragia en un órgano crítico (ej. sistema nervioso central), además de las medidas estándar, requieren rápida restauración de la hemostasia mediante administración de agentes reversores. En pacientes recibiendo dabigatrán, el antídoto específico idarucizumab debe suministrarse a la brevedad, si está disponible. Si idarucizumab no está disponible, CCP-4 o CCPA pueden ser usados. Dado que el andexanet y el ciraparantag aún no se encuentran disponibles en nuestro país, los pacientes recibiendo inhibidores del fXa deben ser tratados con CCP-4 (50 UI/Kg).

En caso de sobredosis, el carbón activado puede reducir la absorción y los niveles plasmáticos de la droga, si esta fue ingerida recientemente $(<2-4 \mathrm{~h})^{49,50}$. La hemodiálisis también es una alternativa en casos de sangrado mayor asociado a dabigatrán en pacientes con deterioro de la función renal, si el idarucizumab no está disponible. En un estudio de 6 pacientes con insuficiencia renal terminal que recibieron una dosis de $50 \mathrm{mg}$ 


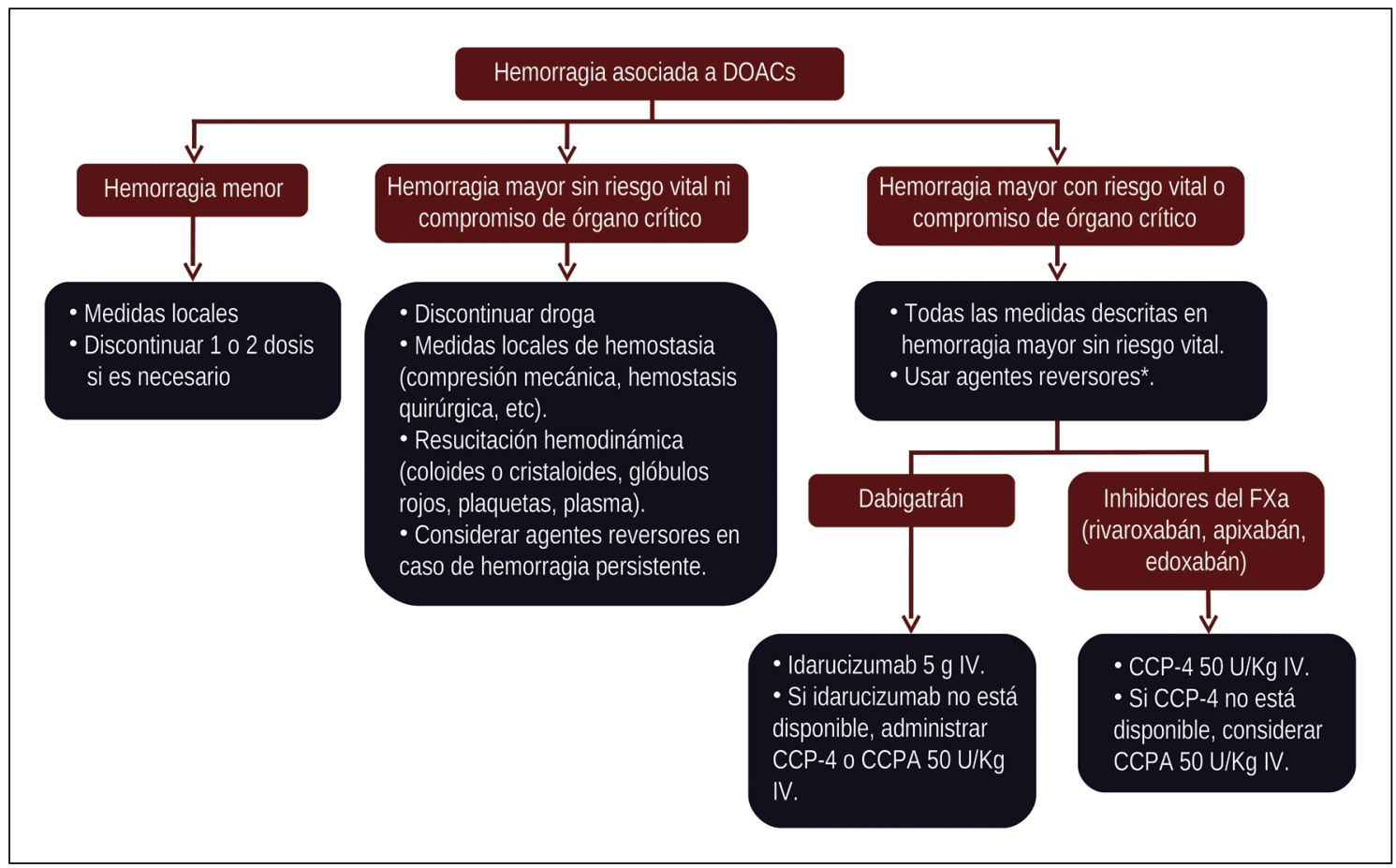

Figura 2. Manejo de hemorragia asociada a DOACs. *Considerar carbón activado en caso de ingestión reciente $(<2-4$ h).

de dabigatrán, $62 \%$ de la droga circulante fue removida a las $2 \mathrm{~h}$ de hemodiálisis y $68 \%$ después de $4 \mathrm{~h}^{51}$. El rivaroxabán, apixabán y edoxabán, debido a su alta unión a proteínas plasmáticas, no son dializables.

\section{Referencias}

1. Ruff CT, Giugliano RP, Braunwald E, Hoffman EB, Deenadayalu N, Ezekowitz MD, et al. Comparison of the efficacy and safety of new oral anticoagulants with warfarin in patients with atrial fibrillation: a meta-analysis of randomised trials. Lancet 2014; 383 (9921): 955-62.

2. Yeh CH, Gross PL, Weitz JI. Evolving use of new oral anticoagulants for treatment of venous thromboembolism. Blood 2014; 124 (7): 1020-8.

3. Connolly SJ, Ezekowitz MD, Yusuf S, Eikelbom J, Oldgren J, Parekh A, et al; RE-LY Steering Committee and Investigators. Dabigatrán versus warfarin in patients with atrial fibrillation. N Engl J Med 2009; 361 (12): 1139-51.

4. Patel MR, Mahaffey KW, Garg J, Pan G, Singer DE, Hac- ke W, et al. Rivaroxabán versus warfarin in nonvalvular atrial fibrillation. N Engl J Med 2011; 365: 883-91.

5. Giugliano RP, Ruff CT, Braunwald E, Murphy SA, Wiviott $\mathrm{SD}$, Halperin JL, et al. Edoxabán versus warfarin in patients with atrial fibrillation. N Engl J Med 2013; 369: 2093-104.

6. Granger CB, Alexander JH, McMurray JJ, Lopes RD, Hylek EM, Hanna M, et al. Apixabán versus warfarin in patients with atrial fibrillation. N Engl J Med 2011; 365: 981-92.

7. Enriquez A, Lip GY, Baranchuk A. Anticoagulation reversal in the era of the non-vitamin K oral anticoagulants. Europace 2016; 18 (7): 955-64.

8. Levy JH, Douketis J, Weitz JI. Reversal agents for non-vitamin $\mathrm{K}$ antagonist oral anticoagulants. Nat Rev Cardiol 2018; 15 (5): 273-81.

9. Cohen AT, Harrington RA, Gibson CM. Betrixaban in Acutely Ill Medical Patients. N Engl J Med 2016; 375 (24): e50.

10. Eriksson BI, Quinlan DJ, Weitz JI. Comparative pharmacodynamics and pharmacokinetics of oral direct thrombin and factor xa inhibitors in development. Clin Pharmacokinet 2009; 48 (1): 1-22. 
11. Raval AN, Cigarroa JE, Chung MK, Díaz-Sandoval LJ, Diercks D, Piccini JP, et al; American Heart Association Clinical Pharmacology Subcommittee of the Acute Cardiac Care and General Cardiology Committee of the Council on Clinical Cardiology; Council on Cardiovascular Disease in the Young; and Council on Quality of Care and Outcomes Research. Management of Patients on Non-Vitamin K Antagonist Oral Anticoagulants in the Acute Care and Periprocedural Setting: A Scientific Statement From the American Heart Association. Circulation 2017; 135 (10): e604-33.

12. Steffel J, Verhamme P, Potpara TS, Albaladejo P, Antz M, Desteghe L, et al; ESC Scientific Document Group . The 2018 European Heart Rhythm Association Practical Guide on the use of non-vitamin K antagonist oral anticoagulants in patients with atrial fibrillation. Eur Heart J 2018; 39 (16): 1330-93.

13. Chang SH, Chou IJ, Yeh YH, Chiou MJ, Wen MS, Kuo CT, et al. Association Between Use of Non-Vitamin K Oral Anticoagulants With and Without Concurrent Medications and Risk of Major Bleeding in Nonvalvular Atrial Fibrillation. JAMA 2017; 318 (13): 1250-19.

14. Atrial Fibrillation Investigators. Risk factors for stroke and efficacy of antithrombotic therapy in atrial fibrillation. Analysis of pooled data from five randomized controlled trials. Arch Intern Med 1994; 154 (13): 1449-57.

15. van der Meer FJ, Rosendaal FR, Vandenbroucke JP, Briët E. Bleeding complications in oral anticoagulant therapy. An analysis of risk factors. Arch Intern Med 1993; 153 (13): 1557-62.

16. Jackson SL, Peterson GM, Vial JH, Daud R, Ang SY. Outcomes in the management of atrial fibrillation: clinical trial results can apply in practice. Intern Med J 2001; 31 (6): 329-36.

17. Hart RG, Diener HC, Yang S, Connolly SJ, Wallentin L, Reilly PA, et al. Intracranial hemorrhage in atrial fibrillation patients during anticoagulation with warfarin or dabigatrán: the RE-LY trial. Stroke 2012; 43 (6): 1511-7.

18. Hylek EM, Held C, Alexander JH, Lopes RD, De Caterina $\mathrm{R}$, Wojdyla DM, et al. Major bleeding in patients with atrial fibrillation receiving apixabán or warfarin: The ARISTOTLE Trial (Apixabán for Reduction in Stroke and Other Thromboembolic Events in Atrial Fibrillation): Predictors, Characteristics, and Clinical Outcomes. J Am Coll Cardiol 2014; 63: 2141-7.

19. Ntaios G, Papavasileiou V, Makaritsis K, Vemmos K, Michel P, Lip GYH. Real-World Setting Comparison of Nonvitamin-K Antagonist Oral Anticoagulants Versus Vitamin-K Antagonists for Stroke Prevention in Atrial Fibrillation: A Systematic Review and Meta-Analysis. Stroke 2017; 48 (9): 2494-503.
20. Cuker A, Siegal DM, Crowther MA, García DA. Laboratory measurement of the anticoagulant activity of the non-vitamin $\mathrm{K}$ oral anticoagulants. J Am Coll Cardiol 2014; 64 (11): 1128-39.

21. Douxfils J, Mullier F, Robert S, Chatelain C, Chatelain $B$, Dogné JM. Impact of dabigatrán on a large panel of routine or specific coagulation assays. Laboratory recommendations for monitoring of dabigatrán etexilate. Thromb Haemost 2012; 107 (5): 985-97.

22. Barrett YC, Wang Z, Frost C, Shenker A. Clinical laboratory measurement of direct factor Xa inhibitors: anti-Xa assay is preferable to prothrombin time assay. Thromb Haemost 2010; 104 (6): 1263-71.

23. Lindhoff-Last E, Samama MM, Ortel TL, Weitz JI, Spiro TE. Assays for measuring rivaroxabán: their suitability and limitations. Ther Drug Monit 2010; 32 (6): 673-9.

24. Neira V, Corbalán R, Pereira J, Panes O, Garayar B, Aizman A, et al. Evaluación de la anticoagulación con rivaroxabán, en pacientes con fibrilación auricular no valvular de reciente diagnóstico. Rev Med Chile 2016; 144: 1103-11.

25. Dentali F, Marchesi C, Giorgi Pierfranceschi M, Crowther M, García D, Hylek E, et al. Safety of prothrombin complex concentrates for rapid anticoagulation reversal of vitamin $\mathrm{K}$ antagonists. A meta-analysis. Thromb Haemost 2011; 106 (3): 429-38.

26. Eerenberg ES, Kamphuisen PW, Sijpkens MK, Meijers JC, Buller HR, Levi M. Reversal of rivaroxabán and dabigatrán by prothrombin complex concentrate: a randomized, placebo-controlled, crossover study in healthy subjects. Circulation 2011; 124: 1573-9.

27. Song Y, Wang Z, Perlstein I, Wang J, LaCreta F, Frost RJA, et al. Reversal of apixabán anticoagulation by four-factor prothrombin complex concentrates in healthy subjects: a randomized three-period crossover study. J Thromb Haemost 2017; 15 (11): 2125-37.

28. Zahir H, Brown KS, Vandell AG, Desai M, Maa JF, Dishy V, et al. Edoxabán effects on bleeding following punch biopsy and reversal by a 4 -factor prothrombin complex concentrate. Circulation 2015; 131 (1): 82-90.

29. Majeed A, Ågren A, Holmström M, Bruzelius M, Chaireti R, Odeberg J, et al. Management of rivaroxabán- or apixabán-associated major bleeding with prothrombin complex concentrates: a cohort study. Blood 2017; 130 (15): 1706-12.

30. Mao G, King L, Young S, Kaplan R. Factor Eight Inhibitor Bypassing Agent(FEIBA) for Reversal of Target-Specific Oral Anticoagulants in Life-Threatening Intracranial Bleeding. J Emerg Med 2017; 52 (5): 731-7.

31. van Ryn J, Ruehl D, Priepke H, et al. Reversibility of the anticoagulant effect of high doses of the direct thrombin 
inhibitor dabigatrán, by recombinant Factor VIIa or activated prothrombin complex concéntrate (abstract 0370). Haematologica 2008; 93 (Suppl 1): 148.

32. Warkentin TE, Margetts P, Connolly SJ, Lamy A, Ricci C, Eikelboom JW. Recombinant factor VIIa (rFVIIa) and hemodialysis to manage massive dabigatrán-associated postcardiac surgery bleeding. Blood 2012; 119 (9): 2172-4.

33. Schiele F, van Ryn J, Canada K, Newsome C, Sepúlveda E, Park J, et al. A specific antidote for dabigatrán: functional and structural characterization. Blood 2013; 121: 3554-62.

34. Glund S, Moschetti V, Norris S, Stangier J, Schmohl $M$, van Ryn J, et al. A randomised study in healthy volunteers to investigate the safety, tolerability and pharmacokinetics of idarucizumab, a specific antidote to dabigatrán. Thromb Haemost 2015; 113 (5): 943-51.

35. Glund S, Stangier J, Schmohl M, Gansser D, Norris S, van Ryn J, et al. Safety, tolerability, and efficacy of idarucizumab for the reversal of the anticoagulant effect of dabigatrán in healthy male volunteers: a randomised, placebo-controlled, double-blind phase 1 trial. Lancet 2015; 386 (9994): 680-90.

36. Glund S, Stangier J, Schmohl M, Moschetti V, Haazen $\mathrm{W}$, De Smet M, et al. Idarucizumab, a specific antidote for dabigatrán: immediate, complete and sustained reversal of dabigatrán induced anticoagulation in elderly and renally impaired subjects. Blood 2014; 124: 344 . abstract.

37. Pollack CV Jr, Reilly PA, van Ryn J, Eikelboom JW, Glund S, Bernstein RA, et al. Idarucizumab for Dabigatrán Reversal - Full Cohort Analysis. N Engl J Med 2017; 377: 431-41.

38. Lu G, De Guzmán FR, Hollenbach SJ, Karbarz MJ, Abe K, Lee G, et al. A specific antidote for reversal of anticoagulation by direct and indirect inhibitors of coagulation factor Xa. Nat Med 2013; 19: 446-51.

39. Ghadimi K, Dombrowski KE, Levy JH, Welsby IJ. Andexanet alfa for the reversal of Factor Xa inhibitor related anticoagulation. Expert Rev Hematol 2016; 9 (2): 115-22.

40. Siegal DM, Curnutte JT, Connolly SJ, Lu G, Conley PB, Wiens BL, et al. Andexanet Alfa for the Reversal of Factor Xa Inhibitor Activity. N Engl J Med 2015; 373 (25): 2413-24.

41. Crowther M, Levy GG, Lu G, Leeds J, Lin J, Pratikhya P, et al. A phase 2 randomized, double-blind, placebo-controlled trial demonstrating reversal of edoxabán-induced anticoagulation in healthy subjects by andexanet alfa
(PRT064445), a universal antidote for factor Xa (fXa) inhibitors [abstract]. Blood 2014; 124: 4269.

42. Connolly SJ, Milling TJ Jr, Eikelboom JW, Gibson CM, Curnutte JT, Gold A, et al; ANNEXA-4 Investigators. Andexanet Alfa for Acute Major Bleeding Associated with Factor Xa Inhibitors. N Engl J Med 2016; 375 (12): 1131-41.

43. Laulicht B, Bakhru S, Lee C, Baker C, Jiang X, Mathiowitz E, et al. Small molecule antidote for anticoagulants (abstract). Circulation 2012; 126: 10021.

44. Bakhru S, Laulicht B, Jiang X, Chen L, Grosso M, Morishima Y, et al. Reversal of Anticoagulant-induced Bleeding in External and Internal Bleeding Models by PER977, a Small Molecule Anticoagulant Antidote (abstract). Circulation 2014; 130: A19361.

45. Hollenbach S, Lu G, De Guzmán F, Tan S, Pratikhya P, Pandey A, et al. Andexanet-alfa and PER977 (Arapazine) Correct Blood Loss in a Rabbit Liver Laceration Model - Only Andexanet Reverses Markers of fXa-mediated Anticoagulation (abstract). Circulation 2014; 130: A14657.

46. Ansell JE, Bakhru SH, Laulicht BE, Steiner SS, Grosso M, Brown K, et al. Use of PER977 to reverse the anticoagulant effect of edoxabán. N Engl J Med 2014; 371: 2141-2.

47. US National Library of Medicine. ClinicalTrials.gov https://clinicaltrials.gov/ct2/show/NCT03172910 (2017).

48. Tomaselli GF, Mahaffey KW, Cuker A, Dobesh PP, Doherty JU, Eikelboom JW, et al. 2017 ACC Expert Consensus Decision Pathway on Management of Bleeding in Patients on Oral Anticoagulants: A Report of the American College of Cardiology Task Force on Expert Consensus Decision Pathways. J Am Coll Cardiol 2017; 70 (24): 3042-67.

49. Suryanarayan D, Schulman S. Potential antidotes for reversal of old and new oral anticoagulants. Thromb Res 2014; 133: S158-66.

50. Wang X, Mondal S, Wang J, Tirucherai G, Zhang D, Boyd RA, et al. Effect of activated charcoal on apixabán pharmacokinetics in healthy subjects. Am J Cardiovasc Drugs 2014; 14 (2): 147-54.

51. Stangier J, Rathgen K, Stahle H, Mazur D. Influence of renal impairment on the pharmacokinetics and pharmacodynamics of oral dabigatrán etexilate: an openlabel, parallel-group, single-centre study. Clin Pharmacokinet 2010; 49: 259-68.

52. Hernández I, Zhang Y, Brooks MM, Chin PK, Saba S. Anticoagulation Use and Clinical Outcomes After Major Bleeding on Dabigatrán or Warfarin in Atrial Fibrillation. Stroke 2017; 48 (1): 159-66. 